\title{
Effects of Millimetric Shifts in Breast Cancer Radiotherapy on the Radiation Dose Distribution
}

\author{
Yusuf Tolga Sanlı ${ }^{1 *}$, Funda Cukurcayır $^{1}$, Fatma Abacigil ${ }^{2}$
}

\begin{abstract}
Background: This study aimed to facilitate decision-making in cases of breast cancer radiotherapy shifts by simulating millimetric shifts and analyzing their effects on dose distribution. Methods: The study included 30 patients with left side breast cancer who were treated with three dimensional conformal radiotherapy (3D-CRT) in the Radiation Oncology Department in Hatay Public Hospital, between January 2013 and April 2015. A treatment plan shifting at three axes with six different measures was simulated. Results: The biggest difference in values was $(+3 \mathrm{~mm}$ shift) $476 \mathrm{cGy}$, with a $7.7 \%$ change for heart and $25.6 \%$ for spinal cord. The shifts in values respectively for CTV min, mean, max were $-4.8 \%, 2.5 \%, 4 \%$. The differences for lymphatic min, mean, max were $21.3 \%, 20.3 \%,-12.2 \%$. Conclusion: The most important thing is not the treatment plan quality, but its practicality. The treatment plan must be practical and its practice must be controlled rigidly.
\end{abstract}

Keywords: Breast cancer - radiotherapy - millimetric shifts - radiation dose distribution

Asian Pac J Cancer Prev, 17 (3), 1197-1199

\section{Introduction}

Patients with breast cancer has a long survival rate after treatment and this disease has become an ordinary one in Turkey and all over the world (Ferlay et al., 2010; Carter et al., 1989). Radiotherapy is an important part of the treatment procedure. Radiotherapy has two parts, namely planning and practice. Physicians and medical physicist work together during the planning part; the technicians play a big role during its practice. The most important part is the correction implementation of plan and practice (Wigg et al., 2001). The practice must be carried out precisely as it has been planned. It is commonly observed that the patients move intrafractions and do not lie down on the treatment table in the same position each day. Technicians control the shifts but cannot make the necessary corrections the plan by 1 or 2 millimeters. There is no significant data about the effects of these milimetric shifts. Correcting the position of all patients is virtually impossible because of time and workload concerns but if the shifts are not corrected the treatment could fail and this could cost the patient's life. Could we overlook some shifts or should we correct them all?

\section{Materials and Methods}

The study included 30 patients with left side breast cancer treated with three dimensional conformal radiotherapy (3D-CRT) in our department between January 2013 and April 2015. The ages of the patients

were between 35 and 66. All patients had similar treatment fields and contoured pattern like RTOG contouring atlases (Gentile et al., 2015). Every patient's treatment plan was $5000 \mathrm{cGy}$ in 25 fractions to breast or chest wall and lymphatic field. Every patient had lymphatic treatment field including supraclavicular fossa and axiller levels. Thirteen of them had breast conserving surgery (BCS) and boost radiotherapy. Boost dose given to $\mathrm{BCS}$ patients was 10Gy. Boost radiotherapy doses were not studied in the analysis. We designed the study by excluding the effects of boost radiotherapy because of the small field and long distance from organs at risk (OAR).

We designed the shifts in three axis same. Six different shifts were calculated by Medical Physicists and Eclipse Planning System was used. Every shift was planned

Table 1. ?? [PLEASE SUPPLY]

\begin{tabular}{lcc}
\hline & & Values \\
\hline Age & & $35-66$ \\
Treatment Fields & Chest Wall & 17 \\
& Breast & 13 \\
Dose & Heart & 442cGy (214-803) \\
& Lung & 686cGy (476-798) \\
& Spinal & 2014cGy (938-3463) \\
& Healthy Breast & 360cGy (94-586) \\
CTVmin & 4263cGy (3857-4614) \\
CTVmean & 5076cGy (5026-5153) \\
CTVmax & 5392cGy (5289-5457) \\
& Lymph min & $4564 c G y(4487-4706)$ \\
& Lymph mean & 4990cGy (4901-5140) \\
Lymph max & 5281cGy (5118-5469) \\
\hline
\end{tabular}

${ }^{1}$ Radiation Oncology,Hatay Public Hospital, ${ }^{2}$ Radiation Oncology, Mustafa Kemal University, Hatay, Turkey *For correspondence: drronkyts@gmail.com 
Table 2. ?? [PLEASE SUPPLY]

\begin{tabular}{lccccccc}
\hline & Original & 1 & -1 & 2 & -2 & 3 & -3 \\
\hline Heart & $442 \%$ & $7.2 \%$ & $6.3 \%$ & $7.5 \%$ & $5.2 \%$ & $7.7 \%$ & $6.6 \%$ \\
Lung & $686 \%$ & $6.3 \%$ & $8.5 \%$ & $4.8 \%$ & $8.6 \%$ & $3.5 \%$ & $11.1 \%$ \\
Spinal Cord & $2014 \%$ & $4.9 \%$ & $1.7 \%$ & $-1.9 \%$ & $-0.5 \%$ & $-9.5 \%$ & $25.6 \%$ \\
Healthy Breast & $360 \%$ & $18.8 \%$ & $18.1 \%$ & $21.4 \%$ & $17.5 \%$ & $20.5 \%$ \\
\hline
\end{tabular}

Table 3. ?? [PLEASE SUPPLY]

\begin{tabular}{|c|c|c|c|c|c|c|c|}
\hline & Original & 1 & -1 & 2 & -2 & 3 & -3 \\
\hline CTVmin & 4263 & $2 \%$ & $1.5 \%$ & $2.5 \%$ & $-4.8 \%$ & $2.6 \%$ & $0.3 \%$ \\
\hline CTVmean & 5076 & $0.6 \%$ & $0.5 \%$ & $0.7 \%$ & $0.3 \%$ & $2.5 \%$ & $0.7 \%$ \\
\hline CTVmax & 5392 & $3.6 \%$ & $3.9 \%$ & $3.9 \%$ & $4 \%$ & $2.7 \%$ & $3.8 \%$ \\
\hline Lym min & 3678 & $16.4 \%$ & $21.3 \%$ & $14.3 \%$ & $-2.3 \%$ & $17.2 \%$ & $8 \%$ \\
\hline Lym mean & 4290 & $18.9 \%$ & $18.5 \%$ & $20.3 \%$ & $-4.3 \%$ & $19.8 \%$ & $16.9 \%$ \\
\hline Lym max & 5281 & $2.5 \%$ & $2.2 \%$ & $1.4 \%$ & $-12.2 \%$ & $5.7 \%$ & $2.4 \%$ \\
\hline
\end{tabular}

Table 4. ?? [PLEASE SUPPLY]

\begin{tabular}{ccccccc}
\hline & 1 & 2 & 3 & -1 & -2 & -3 \\
\hline Dose Change & $8.1 \%$ & $7.9 \%$ & $9.2 \%$ & $8.3 \%$ & $6 \%$ & $9.2 \%$ \\
\hline
\end{tabular}

as $1 \mathrm{~mm}$ difference. $+1 \mathrm{~mm}$ shifts at all axis from the originally planned isocenter, followed by $+2 \mathrm{~mm}$ and $+3 \mathrm{~mm}$ differences. The opposite direction shift as $-1 \mathrm{~mm}$, $-2 \mathrm{~mm}$ and $-3 \mathrm{~mm}$ were also investigated. Finally, we have 7 different dose results and plans. We compared all results with the original plan and search for dose differences. SPSS 17.0 was used for variables and data analyses. Minimum, mean and maximum doses were analyzed for CTV \& Lymphatic volumes. Mean doses for heart and lung, max doses for spinal cord (SC) and healthy breast (HB).

\section{Results}

Patient characteristics are summarized in Table 1. Median age of all patients was 52 years (range: $35-$ 66 years). There was not any significant difference in demographic or dose distribution characteristics. The average dose of original plan for heart was $442 \mathrm{cGy}$. The most different value was ( $+3 \mathrm{~mm}$ shift) $476 \mathrm{cGy}$ and $7.7 \%$ change. The most different value for lung was ( $-3 \mathrm{~mm}$ shift) $762 \mathrm{cGy}$ and $11.1 \%$ change from original dose (686cGy). Average dose of original plan for spinal cord was 2014cGy. $2530 \mathrm{cGy}$ and $25.6 \%$ change for $-3 \mathrm{~mm}$ shift. The shifts differences of OAR from original plan are summarized in Table 2. We analyzed minimum, mean and maximum dose values of CTV and lymphatics summarized in Table 3. The most different values respectively for CTV min, mean, max were $-21.3 \%, 20.3 \%,-12.2 \%$.

\section{Discussion}

We designed $t$ his study to make the decision making process easier in relation to intrafractional moves in order to decrease the workload of oncology departments (Wigg DR and Morgan GW, 2001). Heart volume dose changes were between 5.2\%-7.7. There is no significant difference between consecutive shifts. This data is not helpful to decide whether some of the shifts can be overlooked, but it shows a more important result. If heart dose was high in the treatment plan (Bentzen et al., 2010), utmost care is necessary because even a $1 \mathrm{~mm}$ shift can change the dose more than $7 \%$. But this change can occur if the shift is the same in every fraction of treatment. Just one fraction change was $0.03 \%$.

There was no significant difference in other OAR and Treatment volumes between consecutive shifts. For lung, 3.5\% - 11.1 difference, and for healthy breast $16.9 \%$ - 21.4. Most of the dose changes show us higher doses although analyses show that dose changes could be decreasing or increasing (Redpath AT and Muren LP 2006). Spinal cord dose changes $-9.5 \% 25.6 \%$, it is very variative. That would be about point dose analyses. But we do not know cold spot and hot spot in same place. At one fraction $1.03 \%$ change could occur. If the spinal cord maximum dose at treatment plan was $45 \mathrm{~Gy}$ that would be $45.5 \mathrm{~Gy}$ in one fractional shift. So, it can be said that, if the plan has risky doses for OAR, be more careful and inform your technicians.

Dose changes were higher for lymphatic volume than CTV (chest wall or breast). CTV dose change for $1 \mathrm{~mm}$ shift is $2 \%$ and $13 \%$ for lymphatic volumes. When we turn back to planning software, we discovered that the volumes look similar to spheric design and inside the homogen tissues effect the dose change minimum. That could be the reason for huge change between CTV and lymphatic volume.

Analyzing the shifts we calculate the average of all volumes in consecutive shifts (Table 4). $6 \%$ for $-2 \mathrm{~mm}$ shift was the smallest change. There was a big difference between $-2 \mathrm{~mm}$ and $-3 \mathrm{~mm}$ shift, but that is not a useful data for practice.

When this study was designed a lot of possibilities that would change shifts and dose changes were also taken into account. We could simulate more patient data or other cancer data like prostate, pancreas etc. The most important thing was possible combinations. We designed same value for all axis like $+1 \mathrm{~mm}$ every dimension $+2 \mathrm{~mm}$, and $+3 \mathrm{~mm}$ for every dimension, but this could be randomized like $0 \mathrm{~mm}$ to one axis, $3 \mathrm{~mm}$ for another and $-1 \mathrm{~mm}$ for last one. 342 different combinations are possible, but we chose to 
simulate a few to guess the big picture.

In Conclusion, When we started this study, we planned to decrease the workload of technicians and have a clear conscience at the same time. The study showed that the most important thing is not your treatment plans quality, but its practicality. The treatment plan must be practical and its practice must be controlled rigidly.

\section{References}

Bentzen SM, Constine LS, Deasy JO, et al (2010). Quantitative Analyses of Normal Tissue Effects in the Clinic (QUANTEC): An Introduction to the Scientific Issues. Int J Rad Onc Biol Phys, 1, 76.

Carter CL1, Allen C, Henson DE (1989). Relation of tumor size, lymph node status, and survival in 24,740 breast cancer cases. CANCER, 163, 181-7.

Ferlay J, Hery C, Autier P, et al (2010). Global Burden of Breast Cancer. Breast Cancer Epidemiology, Eds Christopher I.Li. Springer Science Business Media, LLC, 1-19.

Gentile MS, Usman AA, Neuschler EI, et al (2015). Contouring Guidelines for the Axillary Lymph Nodes for the Delivery of Radiation Therapy in Breast Cancer: Evaluation of the RTOG Breast Cancer Atlas. Int J Rad Onc Biol Phys, 93, 257-65.

Redpath AT, Muren LP (2006). CT-guided intensity-modulated radiotherapy for bladder cancer: Isocenter shifts, margins and their impact on target dose. Rad Onc, 81, 276-283.

Wigg DR, Morgan GW (2001). Radiation oncology in Australia: Workforce, workloads and equipment 1986-1999. Australasian Rad, 45, 146-69. 